\title{
ANÁLISIS DEL ESQUEMA DE LA INTEGRAL DEFINIDA DE UN ALUMNO UNIVERSITARIO
}

\section{ANALYSIS OF THE SCHEMA DEFINED INTEGRAL OF A UNIVERSITY STUDENT}

\author{
Eliécer Aldana Bermúdez ${ }^{1}$ Mạ Teresa González Astudillo² \\ ${ }^{1}$ Docente Programa de Licenciatura en Matemáticas. Universidad del Quindío-Armenia, Colombia \\ ${ }^{2}$ Docente investigadora Universidad de Salamanca Paseo de canalejas 169, CP: 37008, España
}

Recibido: Junio 21 de 2010

Aceptado: Noviembre 30 de 2010

Correspondencia: Programa de Matemáticas, Universidad del Quindío, Av. Bolivar calle 12 norte Armenia Quindío. Correo electrónico: eliecerab@uniquindio.edu.co

\section{RESUMEN}

En este artículo se describe cómo se desarrolla el esquema de un alumno universitario que cursa tercer año de Licenciatura de Matemáticas y que ha recibido por primera vez una instrucción previa del concepto matemático de Integral Definida. Como paso inicial de esta investigación se hizo un análisis de libros de texto para identificar los elementos matemáticos que configuran el concepto de Integral Definida, lo que permitió hacer una descomposición genética de dicho concepto. Posteriormente, para recoger los datos, se aplicó primero una prueba piloto a partir de la cual se diseñaron tres instrumentos definitivos: un cuestionario, una entrevista y un mapa conceptual. El análisis de los datos se hizo identificando los elementos matemáticos que utiliza el alumno para resolver las distintas tareas planteadas, las relaciones lógicas que establece entre estos elementos matemáticos y el uso que hace de los sistemas de representación gráfico, algebraico y analítico. Los resultados del análisis nos permitieron caracterizar su nivel de desarrollo del esquema conceptual relativo al concepto de Integral Definida.

Palabras clave: Integral Definida, desarrollo del esquema, elemento matemático, descomposición genética.

\begin{abstract}
This article describes the development of the scheme of the Definite Integral concept of a university student who deals the third year of Bachelor of Mathematics. This is the first time he studies this mathematical concept. In this research, initially was done a textbooks review to identify the mathematical elements that form the Definite Integral concept. This allowed us to design its genetic decomposition. To gather data, firstly a pilot questionnaire was applied, and then three definitive instruments were constructed: a questionnaire, an interview and a conceptual map. The analysis of the data was done identifying the mathematical elements that the student used to solve the different tasks, the logical relations established between these mathematical elements and the use of different systems of representation like graphical, algebraic and analytical systems of representation. The results of the analysis allowed us to characterize its level of development of the conceptual scheme related to the Definite Integral concept.
\end{abstract}

Key words: Definite Integral, development of the scheme, mathematical element, genetic decomposition.

Rev. Invest. Univ. Quindío (21): 95- 107. Armenia - Colombia 


\section{INTRODUCCIÓN}

Muchos han sido los investigadores que han señalado las dificultades de los alumnos en relación con el aprendizaje y la comprensión del concepto de integral definida. Una de los primeros en comprobar algunas de estas dificultades es Orton (1983) quien observa que los alumnos son capaces de realizar cálculos algebraicos en los que intervienen las integrales pero no son capaces de comprender el papel que juega el límite en la definición de este concepto y no son capaces de dotar de significado a los símbolos que se utilizan en estos cálculos. En otras investigaciones se han identificado dificultades para calcular ciertas integrales como $\beta_{-3}^{3} x+2 d x$ (Mundy, 1984), la identificación entre el concepto de Integral Definida y el cálculo de áreas (Clavo, 1997), o la incoherencia entre la imagen del concepto y la definición del concepto (Rasslan y Tall, 2002). Contreras y Ordóñez (2005) hicieron un análisis de los significados personales de los estudiantes utilizando como marco teórico el Enfoque Ontológico Semiótico (EOS) de la cognición matemática (Godino, 2002) con el que pudieron concluir que los estudiantes demuestran confusión entre la Integral Definida y el cálculo del área; tienen dificultades para argumentar o justificar sus respuestas; presentan conflictos semióticos para trabajar el concepto de Integral Definida en contextos distintos de las propias matemáticas como es el caso del movimiento uniforme de la Física; ponen de manifiesto conflictos asociados a la elección errónea de los límites de integración, y algunos confunden la Integral con la derivada; tienen dificultades para manejar el lenguaje gráfico; y sólo son capaces de alcanzar un mayor grado de comprensión de algunos conceptos cuando, además de haber adquirido sus significados, son capaces de transferirlos a otros contextos.

Para describir las concepciones de los alumnos en torno a este concepto, Dubinsky et al (2000) realizaron un análisis epistemológico de las aportaciones de matemáticos tales como Arquímedes, Cavalieri, Wallis y Roberval al desarrollo de este concepto, concluyendo que, en relación con el cálculo de la integral definida, existen dos puntos de vista distintos: o bien subdivisiones asociadas al método de exhausción de los griegos que luego fue refinado por el método de aproximación de las sumas de Riemann, o bien el método de los indivisibles, que implica considerar, por ejemplo, una superficie como compuesta por líneas paralelas. A partir de este estudio histórico se compararon estas concepciones de los matemáticos con las justificaciones ofrecidas por estudiantes universitarios en una entrevista después de una instrucción previa concluyendo que había algunas similitudes entre ambas y que, además, los estudiantes mostraban una visión diferente a la desarrollada durante la instrucción recibida.

Ahondando en este tema y tratando de comprender cómo se llega a adquirir este concepto, en una investigación posterior, Czarnocha et al., (2001), muestran que para la comprensión del concepto de Integral Definida es necesaria la coordinación de dos esquemas, el esquema visual de la suma de Riemann, de índole geométrico, y el esquema de los límites de la secuencia numérica, de índole aritmético. En este mismo sentido, Paschos et al. (2006) aborda la abstracción reflexiva en la construcción del concepto de Integral Definida, con una alumna universitaria.

Para medir el grado de comprensión de la Integral Definida Boigues y Pastor (2007) utilizaron la teoría Fuzzy (George y Veeramani, 1994) para cada estudiante analizado concluyendo que el grado de desarrollo global respecto al esquema de Integral Definida es muy bajo.

A partir del conocimiento de estas dificultades y de los mecanismos de construcción del concepto se han diseñado distintas estrategias metodológicas de enseñanza del concepto de Integral como la de Turégano (1994) que propone como alternativa enseñarla utilizando la génesis histórica del concepto, comenzado con el concepto de integral de forma independiente de la diferenciación y previo al concepto de límite. Utilizando las nuevas tecnologías Depool (2004) diseña diversas situaciones de enseñanza para lograr que los alumnos adquieran la compresión de este concepto a partir de un modelo de competencia cognitivo de la Integral Definida y Camacho et al. (2008) utilizan software adecuado en un curso de ingeniería para ayudar a los alumnos a comprender los conceptos de partición, refinamiento, aproximación y límite.

Rev. Invest. Univ. Quindío (21): 95- 107. Armenia - Colombia 
También hemos de destacar la ingeniería didáctica desarrollada por González-Martín (2006) para mejorar la comprensión del concepto de Integral impropia por parte de los estudiantes de los primeros cursos universitarios.

\section{MARCO TEÓRICO}

Esta investigación trata abordar el problema relativo al aprendizaje del concepto de integral definida siendo nuestro principal objetivo describir el desarrollo de los esquemas de alumnos universitarios de acuerdo con la teoría APOE a partir de una descomposición genética inicial del concepto. El marco teórico en el que se ha desarrollado esta investigación es la teoría desarrollada por Dubinsky (1991) y un grupo de investigadores Research in Undergraduate Mathematics Education Community (RUMEC) a partir de la noción de abstracción reflexiva (Piaget y García, 1982) modificándola para ser aplicada al Pensamiento Matemático Avanzado. Desde esta perspectiva teórica del conocimiento matemático, Dubinsky (1991, 2000a) y Asiala et al., (1996) consideran que los sujetos realizan ciertas construcciones mentales para comprender los conceptos matemáticos. Estas construcciones mentales se denominan: acciones, procesos, objetos y esquemas y pueden ser descritas de la siguiente forma (Asiala et al, 1996):

- Acción: transformación de un objeto que es percibida por un sujeto como algo externo.

- Proceso: interiorización de una acción cuando esta se repite y el individuo reflexiona sobre ella.

- Objeto: encapsulación de un proceso

- Esquema: colección de acciones, procesos, objetos y otros esquemas que están relacionados consciente $o$ inconscientemente en la mente de un individuo en una estructura coherente y que pueden ser empleados en la solución de una situación problemática que involucre esa área de las matemáticas.

Para lograr progresar en estas construcciones mentales hay que desarrollar ciertos mecanismos como: interiorización, coordinación, inversión, encapsulación, desencapsulación, y tematización (Dubinsky, 1991):

- Interiorización: construcción mental de un proceso relativa a una serie de acciones sobre objetos cognitivos.

- Coordinación: a partir de dos o más procesos se construye un nuevo proceso

- Inversión: deshacer un proceso para construir uno nuevo inverso del primero.

- Encapsulación: transformación de un proceso en un objeto

- Tematización: reflexión como un esquema considerado como un todo y siendo capaz de realizar acciones sobre dicho esquema

El refuerzo de la teoría APOE con los tres niveles de desarrollo del esquema propuestos por Piaget $y$ García (1982), ha llevado a mejorar la comprensión y explicación del concepto de esquema (Dubinsky y MacDonalds, 2001). DeVries (2001), caracteriza los niveles de desarrollo de un esquema como:

- Intra, cuando sólo se identifican aspectos individuales aislados

- Inter, se caracteriza por la construcción de relaciones y

- Trans, se adquiere cuando se tiene construida una estructura completa, las relaciones descubiertas en el ínter son comprendidas dando coherencia al esquema.

En opinión de Baker et al., (2000), el uso de estos niveles para analizar el conocimiento de los estudiantes ayuda a los investigadores a considerar la riqueza de las situaciones y de los problemas de investigación.

Esta idea fue matizada en el estudio de SánchezMatamoros (2004) sobre el desarrollo de la comprensión del concepto de derivada quien completó los niveles de desarrollo mediante subniveles para diferenciar los diferentes esquemas de los alumnos. Para caracterizar dichos subniveles se analizan los elementos matemáticos y las relaciones lógicas entre ellos que utilizan los alumnos en la realización de tareas. Las relaciones lógicas (conjunción lógica, contrarecíproco y equivalencia lógica) se entienden como 
"coordinación entre operaciones" que se establecen entre los elementos matemáticos cuando se resuelve un problema y los elementos matemáticos como "el producto de una disociación o de una segregación en el interior de una totalidad previa".

\section{METODOLOGÍA}

Esta investigación forma parte de un estudio más amplio desarrollado en la Universidad del Quindío para caracterizar el desarrollo del esquema de Integral Definida de los alumnos que cursan tercer año (sexto semestre) de Licenciatura de Matemáticas y que estudian por primera vez el concepto de Integral Definida.

Para realizar esta investigación se llevó a cabo inicialmente una revisión de diferentes libros de texto que incluían el concepto de Integral Definida para determinar los elementos matemáticos que configuran este concepto matemático. Dichos elementos se clasificaron en los siguientes apartados: el área como aproximación (ACA); el área como límite de una suma (ALS); la Integral Definida (LID), las propiedades de las integrales (PID) y los teoremas fundamentales y del valor medio (TFV), desde los sistemas de representación gráfico $(G)$, algebraico (A) y analítico (AN), a partir de estos elementos matemáticos se estableció una descomposición genética previa de dicho concepto que permitiera analizar las producciones de los alumnos.

Posteriormente, se diseñó un precuestionario que fue validado por expertos en Didáctica del Análisis españoles y que fue aplicado de forma experimental. A partir del informe de los expertos y de los resultados de los alumnos se elaboró el cuestionario definitivo que constaba de ocho tareas ${ }^{1}$ y que fue contestado por once alumnos. Además se estableció el guión de una entrevista semiestructurada (Ginsburg et al., 1983) para poder describir y explicar de forma detallada el nivel de desarrollo del esquema de Integral Definida de cada alumno. Dichas entrevistas fueron audiograbadas. Finalmente, para completar la información y con el objetivo fundamental de la triangulación de los

\footnotetext{
${ }^{1}$ Las distintas tareas del cuestionario se pueden ver en el apartado correspondiente a los resultados.
}

instrumentos utilizados, los alumnos realizaron un mapa conceptual sobre el concepto de Integral Definida.

El análisis de los datos se realizó utilizando conjuntamente los resultados recogidos a través de los tres instrumentos mencionados anteriormente: el cuestionario, la entrevista y el mapa conceptual. Para describir el desarrollo del esquema de cada alumno se consideraron los niveles y subniveles: INTRA 1, INTRA, INTER 1, INTER y TRANS, (SánchezMatamoros, 2004) caracterizados por las relaciones lógicas que un sujeto es capaz de establecer entre los diferentes elementos matemáticos y por la coordinación entre los diferentes sistemas de representación: gráfico (G), algebraico (A) y analítico (AN) en la resolución de las distintas tareas. Estos niveles se construyen de manera progresiva, continua y ascendente.

\section{RESULTADOS Y DISCUSIÓN}

Vamos a describir el desarrollo del esquema del concepto de Integral Definida de uno de los alumnos que participaron en este estudio. Para ello vamos a presentar la producción de este alumno en relación con las tareas de cuestionario completándola con sus intervenciones a lo largo de la entrevista.

El desarrollo del esquema de este alumno está caracterizado porque no es capaz de establecer relaciones lógicas entre los elementos matemáticos, es decir, utiliza algunos elementos matemáticos de forma aislada. Por ejemplo, en la tarea 1 :

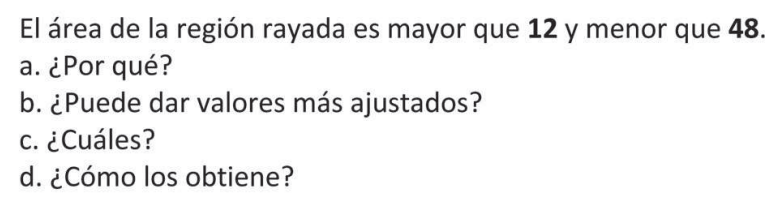

El área de la región rayada es mayor que 12 y menor que 48.

a. ¿Por qué?

b. ¿Puede dar valores más ajustados?

c. ¿Cuáles?

d. ¿Cómo los obtiene?

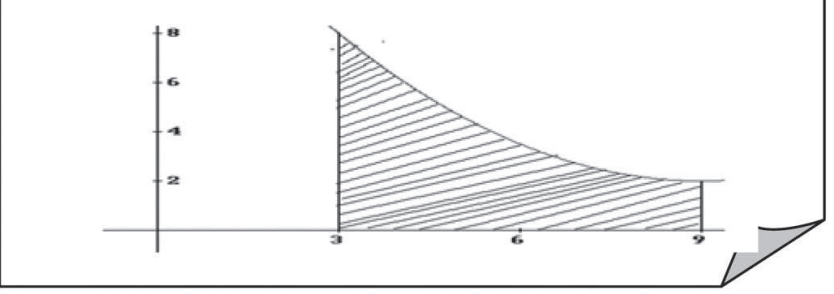

Rev. Invest. Univ. Quindío (21): 95- 107. Armenia - Colombia 
Representa gráficamente la función calculando una aproximación gráfica por exceso y por defecto del área bajo la curva mediante figuras geométricas apropiadas para ajustar el área.

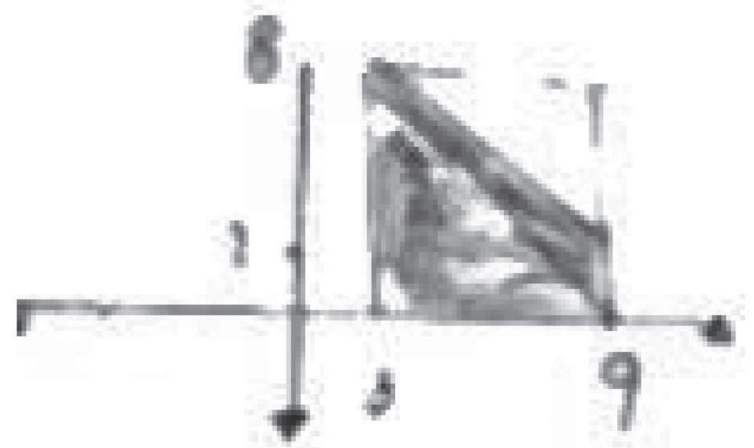

Figura 1. Representación G de la tarea 1 del cuestionario

Es decir, utiliza el elemento matemático ACA de forma G, porque forma un rectángulo de base el segmento determinado por los extremos en el eje de abscisas entre los que se quiere calcular el área y de altura la ordenada correspondiente a la abscisa 3. Traza su diagonal para formar dos triángulos rectángulos, uno de los cuales cubre parte del área sombreada. Sobre la hipotenusa de éste traza otro triángulo, tratando de cubrir el resto del área sombreada.

Para calcular la aproximación del área por exceso de forma A, a partir de la construcción gráfica realizada, calcula el área del rectángulo y luego da una aproximación restando cierta cantidad $(x=20)$ que él considera adecuada, como se puede ver en la siguiente imagen:

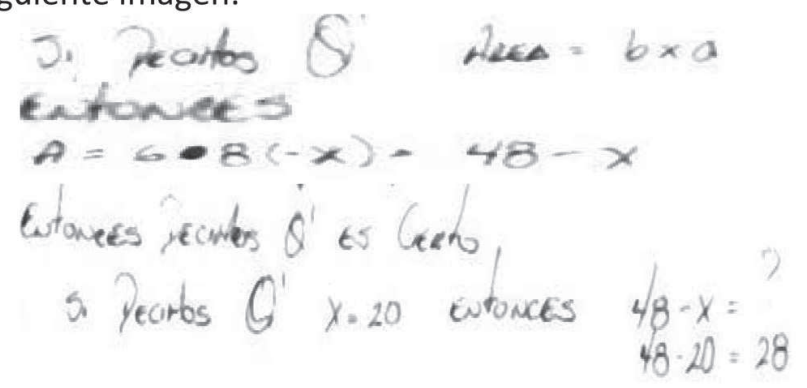

Figura 2. Resolución A de la tarea 1 del cuestionario

Está utilizando un único elemento matemático ACA de forma $\mathbf{A}$, porque calcula el área del rectángulo a partir de su fórmula, llama $x$ a la región que está por encima de la sombreada, le da por tanteo el valor de 20 y calcula el valor aproximado del área bajo la gráfica como 28 unidades cuadradas.

Rev. Invest. Univ. Quindío (21): 95- 107. Armenia - Colombia
Durante la entrevista reconoce que no es capaz de hacerlo de otra manera:

A5: Decimos que el área es igual a base por altura, entonces armamos el cuadro y tratamos de hacer una línea para hallar el área.

I: ¿Por qué le resta $x$ ?

A5: Porque, no sabemos cuánto vale el área que tenemos en blanco, con la que formamos el cuadro, para hallar los otros valores.

I: ¿Por qué justifica las cotas de esa forma?

A5: No, son valores que uno le da como para hacerlos.

I: ¿Existe otra forma de ajustar las cotas?

A5: Posiblemente por sumatorias de Riemann, pero no sé.

I: ¿Cómo sería por una sumatoria de Riemann?

A5: No, tocaría hallarle el área a cada uno, pero no, eso es complicado, pero sinceramente en este momento no me acuerdo.

Aunque en este extracto, menciona un cuadro, en realidad se está refiriendo al rectángulo que ha construido de forma gráfica de base 6 unidades y de altura 8 unidades. Como puede notarse, finalmente el estudiante no recurre al área de los triángulos que ha formado, ni del trapecio que trazó sobre la región sombreada para aproximarla.

Por otra parte, cuando se le pregunta por otras formas de aproximación recuerda el nombre de las sumas de Riemann, pero no es capaz ni siquiera de plantearlas.

Otra tarea en la que el alumno utiliza el mismo elemento matemático y de forma similar es la tarea 3:

Sea $\mathbf{R}$, la región entre la gráfica de la función $f(x)=x^{2}$ y el eje $x$, en el intervalo $[0,4]$.

Utilizar particiones para aproximar el valor del área de la región $\mathbf{R}$ y justificar la respuesta.

Este alumno representa inicialmente de forma $\mathbf{G}$ la función, luego está utilizando el elemento matemático ACA de forma G: 


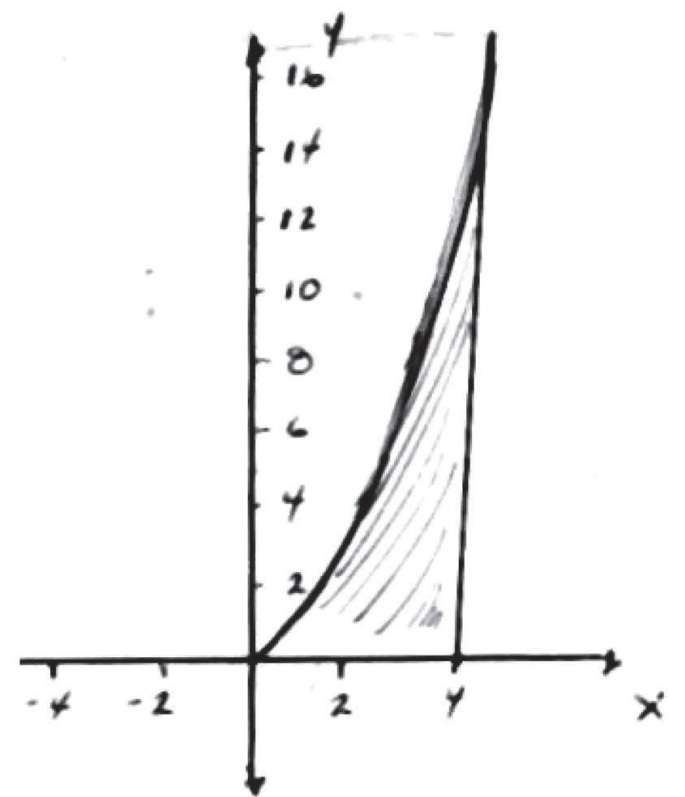

Figura 3. Representación $\mathrm{G}$ de la tarea 3 del cuestionario

Pero para calcular una aproximación recurre a una representación $\mathbf{A}$ de dicho elemento matemático como se puede observar a continuación sin tener en cuenta la representación gráfica previamente realizada:

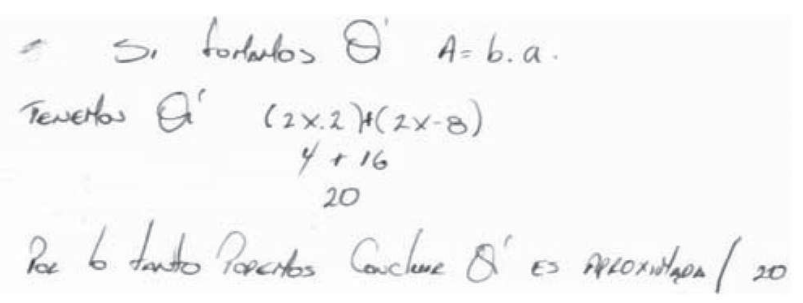

Figura 4. Resolución A de la tarea 3 del cuestionario

Para ello subdivide el intervalo en dos subintervalos de longitud de la base 2 unidades cada uno. Forma dos rectángulos, uno de altura 2 y otro de altura 8 y aplica la fórmula del área correspondiente para aproximar el área. No hay coordinación entre los cálculos algebraicos y la representación gráfica puesto que las alturas no se corresponden con ninguna figura geométrica que haya representado en la gráfica y tampoco se corresponden con los puntos por los que pasa la curva (las alturas de los rectángulos deberían haber sido 4 y 16). Durante la entrevista, cuando se le pregunta por los cálculos que realizó en el cuestionario, no es capaz de establecer un razonamiento lógico que los justifique.
I: ¿La expresión $2 \times 2$ de dónde la obtiene?

A5: Porque ésta es la base que sería...

I: ¿Qué quiere decir con que ésta es la base?

A5: Que queremos hallar el área y necesitamos saber cuál es la base de la figura que nos dan, entonces decimos que $2 \times 2$.

I: ¿De dónde obtiene $2 \times 2+2 \times 8$ ? ¿De dónde obtuvo ésta expresión?

A5: Tiene que ser de la figura.

I: ¿Cómo de la figura, cómo la obtuvo, de dónde la sacó, qué haría en este momento?

A5: No, no me acuerdo profe.

Se pone de manifiesto que utiliza los conocimientos que tiene sobre el concepto de función para graficar la función cuadrática, recuerda la fórmula del área del rectángulo y utiliza un cálculo numérico erróneo para aproximar el área, que no sabe justificar, y no coordina los sistemas de representación gráfico y algebraico por lo que se puede inferir que este alumno sólo menciona este elemento matemático de manera aislada sin coordinar los diferentes sistemas de representación.

En general, este alumno se siente más cómodo cuando trabaja en el registro algebraico que en el registro gráfico. Esto se puede observar claramente cuando se le pide que realice un razonamiento gráfico en la tarea 6:

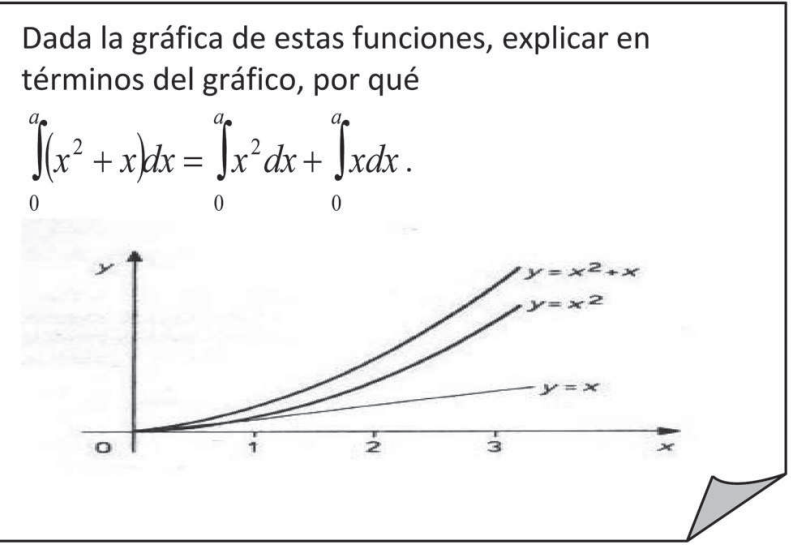

Utiliza un procedimiento exclusivamente algebraico para resolverla, a pesar de que la tarea dice que se explique la propiedad en términos gráficos. 


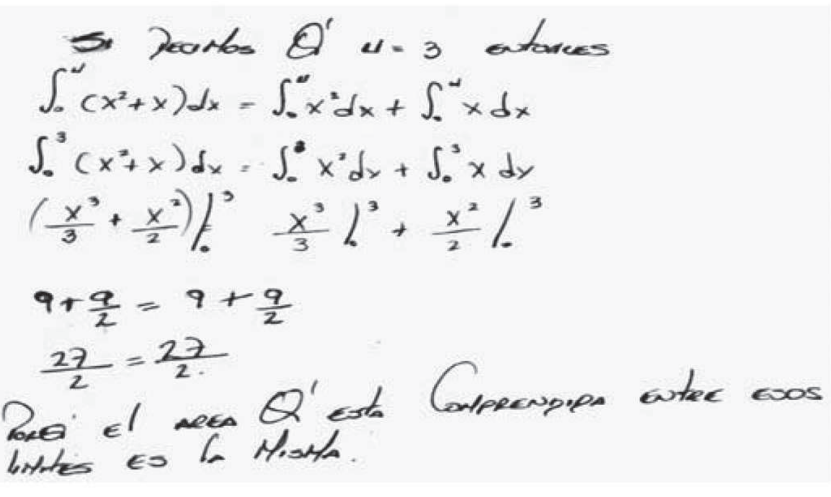

Figura 5. Resolución A de la tarea 6 del cuestionario

Por la forma como responde se pone de manifiesto que recurre al elemento matemático el teorema fundamental del Cálculo (TFC) de forma A, porque para demostrar la propiedad de linealidad se siente obligado a calcular la integral en el intervalo $[0,3]$ aplicando la regla de Barrow a ambos lados de la igualdad. Se limita a desarrollar las integrales propuestas en el ejercicio, y como no es capaz de hacerlo cuando uno de los límites del intervalo de integración viene dado por una letra (a), lo sustituye por un número que relaciona con la gráfica (ya que 3 es el último número que aparece en la escala del eje de abscisas) y comprueba que obtiene el mismo resultado numérico a ambos lados de la igualdad.

Durante la entrevista él mismo señala que no es capaz de justificar la propiedad de forma $\mathbf{G}$.

I: ¿Podría explicarme que quiere decir con este razonamiento?

A5: Aquí, nos dan las integrales en unos intervalos, pero el intervalo superior está limitado por $u$, entonces reemplazamos.

I: Es de cero a a, todos tienen el mismo intervalo de cero a $a$.

A5: Lo tomé como $u$, porque no se ve bien, entonces le damos un valor más o menos aproximado a la gráfica y lo resolvemos.

I: ¿Podría demostrarlo gráficamente?

A5: No

Además, por alguna deficiencia en las fotocopias, no es capaz de distinguir el extremo superior del intervalo de integración (a) al que cambió la denominación y lo llamó $u$ y, como reconoce que no puede manejar la integral como un objeto, le da un valor numérico a esta variable (3) y luego calcula las integrales que constituyen la propiedad para demostrarla, obteniendo una igualdad numérica; no logra utilizar los elementos matemáticos necesarios para demostrar la propiedad de la unión de intervalos de forma $\mathbf{G}$.

Otra característica determinante de este alumno consiste en recordar algún elemento matemático a lo largo de todo el cuestionario, vinculado sólo a un sistema de representación, gráfico, algebraico o analítico. Como hemos mencionado, este alumno tiene cierta tendencia a trabajar exclusivamente en el registro algebraico sin coordinarlo con la representación gráfica lo que le conduce a ciertos conflictos. Esto se pone de manifiesto en la manera que tiene este alumno de resolver la tarea 2 :

Sea $\mathbf{R}$, la región encerrada por el gráfico de la función $f(x)=4 x$ y el eje $x$, en el intervalo $[-2,2]$.

a. Dibujar la gráfica

b. Calcular gráficamente el área de la región $\mathrm{R}$.

c. Calcular la $\int_{-2}^{2} 4 x d x$

d. ¿Son iguales los dos resultados anteriores? Justificar cada paso.

En la respuesta al cuestionario, este alumno lo primero que hace es representar gráficamente la función, con lo que responde correctamente al primer apartado de esta pregunta:

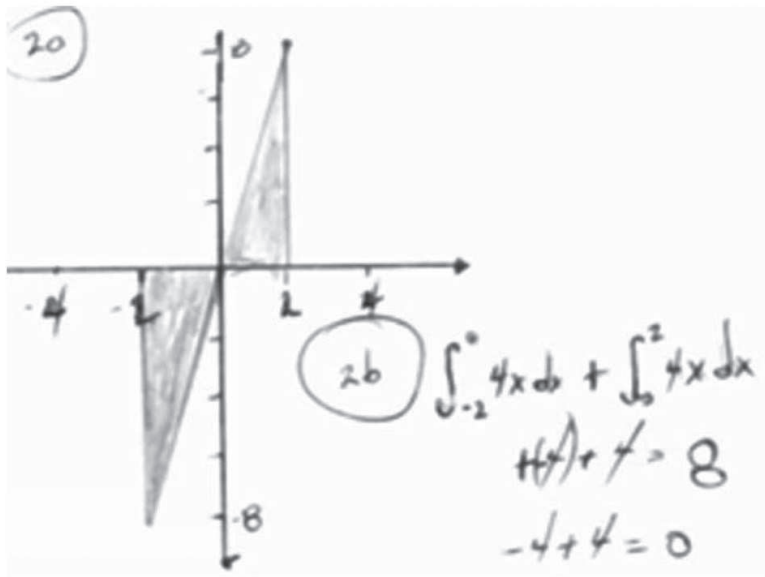

Figura 6. Resolución G y A de la tarea 2 del cuestionario

Para calcular el área no es capaz de utilizar ACA de forma G, como se le pide en el segundo apartado, mediante el cálculo del área de los triángulos que se han formado y recurre al elemento matemático TFC como cálculo algebraico aunque de forma incorrecta 
porque aplica mal la regla de Barrow y no se da cuenta que el valor del área no puede ser cero. Cuando trata de calcular la integral lo hace exclusivamente de forma $\mathbf{A}$.

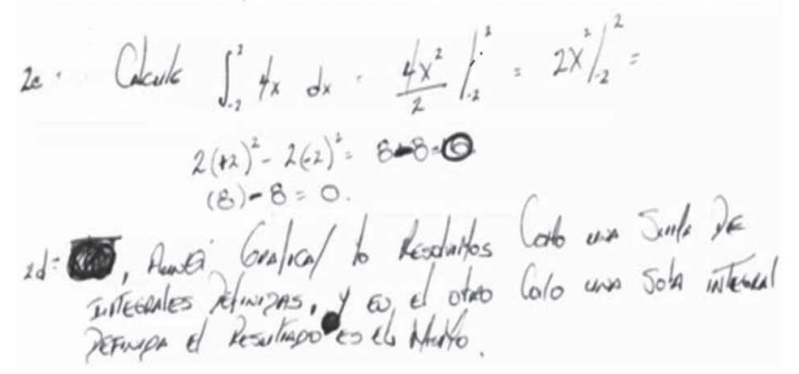

Figura 7. Resolución A de la tarea2 del cuestionario

Para ello, aplica el elemento matemático TFC utilizando la regla de Barrow y cómo no fue capaz de utilizar LID como área de una región obtiene el mismo resultado anterior, entonces afirma que la Integral Definida como cálculo algebraico es lo mismo que la Integral como área de una región. Estos son los argumentos que utiliza cundo justifica el procedimiento anterior.

\section{I: ¿Cómo calculó el área gráficamente?}

A5: Por eso, por una integral.

I: ¿Qué le piden aplicar la integral o calcular gráficamente?

A5: No, aplicar gráficamente, hallar el área de éste triángulo y restarle éste.

I: ¿Cómo sería?

A5: Con la Integral Definida.

I: ¿El área de un triángulo se haya con la Integral Definida?

A5: No, no me acuerdo, porque es que aquí me dice calcular gráficamente la región.

I: ¿Qué valor obtuvo al calcular la integral?

A5: Supuestamente cero.

I: ¿'Por qué da cero la integral?

A5: Porque al evaluarlo primero en la parte superior y en la inferior me daría cero.

I: ¿Un área puede dar cero?

A5: No...

I: ¿Cómo calcularía el área?

A5: No, no me acuerdo profe.

En el protocolo de la entrevista pone de manifiesto que no es capaz de utilizar el elemento matemático ACA de forma gráfica, de hecho señala que para calcular el área de la región sombreada hay que restar el área de los dos triángulos formados por la gráfica. Se limita a utilizar los elementos matemáticos de forma algebraica tanto para calcular la integral como el área, sin coordinar con los aspectos algebraicos con la representación gráfica y obtiene como resultado cero. Él es consciente que el área no puede ser cero y ésta situación le genera un conflicto.

En tercer lugar este alumno recuerda algunos elementos matemáticos con errores en las tareas 4, 5 , 7c y 8 . Sus errores están relacionados con cálculos incorrectos (relacionados con los signos que se obtienen al aplicar la regla de Barrow), el desconocimiento de la función valor absoluto, el cálculo incorrecto de la primitiva de alguna función, su falta de capacidad en el manejo del registro gráfico, el desconocimiento de las condiciones para aplicar la regla de Barrow y concepciones erróneas de la Integral Definida y de las sumas de Riemann. Por ejemplo, en la tarea 4:

Calcular el área limitada por la gráfica de la función $f(x)=|2 x-1|$, en el intervalo

$[0,2]$ y el eje $x$. Justificar la respuesta.

El alumno no realizó ninguna gráfica de la función por lo que responde de forma exclusivamente $\mathbf{A}$ en el cuestionario:

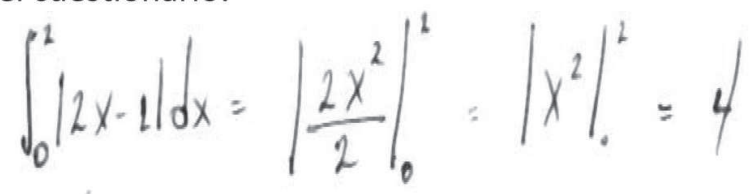

Figura 8. Resolución A de la tarea 4 del cuestionario

En esa respuesta, se puede observar que el alumno, por un lado, no tiene en cuenta que la función que está integrando es la función valor absoluto y prescinde de dicho valor absoluto. Por otra parte, la primitiva asociada a esa función no es correcta, sólo ha integrado una parte de la función, no ha tenido en cuenta el -1 de la función. Los errores están asociados tanto a su desconocimiento de la función valor absoluto como al cálculo de la primitiva. Además, como no ha representado gráficamente la función, no tiene otros recursos para calcular el área que se le pide y en la entrevista no es capaz de desarrollar una justificación a los cálculos que hace:

Rev. Invest. Univ. Quindío (21): 95- 107. Armenia - Colombia 
I: ¿Considera qué esa función está bien integrada? A5: Pues no se profe, en este momento no se, pues yo la hice así pero...

I: ¿Si le piden calcular el área, por qué aplicó la integral?

A5: ¿Si me piden calcular el área?

I: Si le piden calcular el área de una función valor absoluto ¿Por qué aplica la integral de esa función? A5: Porque con ella también la puedo hallar.

Al igual que hizo en la tarea 2, este alumno demuestra que cuando se le pide el cálculo de un área inmediatamente lo asocia con la Integral Definida de memoria sin darse cuenta que podría haber formas más sencillas para calcular esas áreas cuando no se sabe calcular la primitiva de una función o cuando el área formada es simplemente una forma geométrica sencilla de la que se tiene una fórmula algebraica para calcular su área.

También comete algunos errores en la resolución de la tarea 5 cuando tiene que utilizar las propiedades de la integral definida de funciones positivas $y$ negativas.

Dada la gráfica de $f(x)=2 x-x^{2}$, calcular por aproximaciones el área de la región rayada. Justificar el procedimiento utilizado.
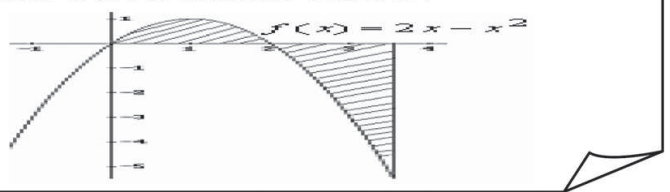

Para responder a esta tarea, el alumno no lo hace mediante aproximaciones sino que se limita a aplicar la regla de Barrow

$$
\begin{aligned}
& \int_{-}^{2} 2 x-x^{2} d x-\int_{0}^{+} 2 x-x^{2} d x \\
& \int_{0}^{2} 2 x-x^{2} d x-\int_{2}^{2} 2 x-x^{2} d x \\
& x^{2}-\left.\frac{x^{3}}{3}\right|_{-} ^{2}-x-\left.\frac{x^{3}}{3}\right|^{+} \\
& 4-\frac{8}{3}-\left[4-\frac{8}{3}-\left(16-\frac{64}{3}\right)\right] \\
& \frac{4}{3}-\left[\frac{4}{3}-\frac{48-6 x}{3}\right] \\
& \frac{4}{3}-\left[\frac{4}{3}+\frac{12}{3}\right] \\
& \frac{4}{3}-\left[\frac{16}{3}\right] \\
& \frac{4}{3}-\frac{16}{3}=-\frac{12}{3}
\end{aligned}
$$

Figura 9. Resolución A de la tarea 5 del cuestionario
Por la forma como responde pone de manifiesto que utiliza el elemento matemático TFC (concretamente la regla de Barrow) y se limita a realizar un cálculo algebraico que le da como resultado un número negativo que no puede ser el área geométrica de la región. Este alumno no ha aplicado correctamente la regla de Barrow al sustituir en los extremos de los intervalos de integración, no ha utilizado el signo menos correctamente y por eso el resultado es un número negativo. Además no utilizó el paréntesis necesario para indicar cuál era la función que estaba en el integrando en cada integral y supuso que el extremo derecho del segundo intervalo de integración era 4 cuando en la gráfica se indica el 3,5 .

Durante la entrevista menciona la propiedad del cálculo de áreas de funciones no estrictamente positivas.

A5: ...hay una fórmula que dice que cuando uno tiene dos áreas hay que mirar la que está por arriba y la que está por debajo, entonces se tiene la que está por arriba y se le resta la que está por debajo y se evalúa en los intervalos dados.

I: ¿Por qué se resta?

A5: Porque está por debajo, si no estoy mal, es porque está por debajo.

I: ¿Siempre que esté por debajo se resta? ¿Cuál es ese criterio?

A5: ¿Cuál es el criterio? No.

I: ¿De qué forma podría aproximar el área?

A5: ¿De qué otra forma lo puedo hacer? Pues con particiones, con una suma de Riemann, pero no.

I: ¿Cómo sería con particiones? ¿Podría comentarlo?

A5: (El estudiante se ríe), los divide en rectángulos y empieza hallar el área.

En el protocolo se pone de manifiesto que menciona una relación entre los elementos matemáticos ACA y TFC; pero, por un lado no es capaz de dar una aproximación del área utilizando sólo el elemento matemático ACA de forma $\mathbf{G}$ ni $\mathbf{A}$ y, por otro, cuando calcula el área usando la regla de Barrow lo hace de forma errónea al confundirse con los signos.

También comete errores cuando trata de resolver la tarea 7c:

Rev. Invest. Univ. Quindío (21): 95- 107. Armenia - Colombia 
Considerar el valor de verdad o de falsedad de la afirmación $\int_{-1}^{1} x^{-2} d x=\left[-x^{-1}\right]_{-1}^{1}=(-1)-1=-2$

y en caso de ser falsa, explicar por qué o dar un contraejemplo.

En el cuestionario responde de forma exclusivamente algebraica mediante el siguiente razonamiento.

$$
\int_{-1}^{2} x^{-2} d x=\left.\left(-x^{-1}\right)\right|_{-1} ^{1}=-\frac{1}{x^{\prime}}=2
$$

Figura 10. Resolución A de la tarea 7c del cuestionario

El procedimiento algebraico que utiliza para resolver la tarea pone de manifiesto que desconoce las condiciones necesarias para poder aplicar la regla de Barrow, porque encuentra la primitiva de la función y la evalúa en los límites de integración, pero no se da cuenta que la función tiene una discontinuidad en un punto del intervalo; es decir desconoce las condiciones para poder aplicar los elementos matemáticos que debe utilizar para justificar el valor de falsedad de la proposición.

En la entrevista insiste en que el procedimiento de solución es correcto aunque la solución final no sea igual a la que él obtuvo:

I: ¿Qué valor le dio a la proposición 7c y por qué?

A5: Dicen que va de -1 a 1, dije que era falsa.

I: ¿Por qué la considera falsa?

A5: Porque la resolvi, no sé, si está bien, me dio lo contrario, un número contrario a la respuesta anterior que nos dan.

I: ¿Qué aplicaron ahí, para resolver esa integral?

A5: En lo que hemos visto, en la integral, se integra la función, la evaluamos en los intervalos, que es -1 y 1 y solucionamos.

I: ¿Está de acuerdo con el procedimiento más no con la respuesta?

A5: No, la respuesta supuestamente es falsa, porque me dio lo contrario es por el procedimiento.

Asocia el concepto de integral definida con un algoritmo para calcularla sin tener en cuenta las condiciones para poder aplicarlo y por eso considera válido el procedimiento, pero la solución final no es igual a la suya porque comete un error en el signo de la respuesta y por tanto piensa que la afirmación es falsa por esa diferencia en el signo. Este alumno desconoce los elementos matemáticos necesarios para poder tomar una decisión sobre la resolución de esta tarea.

Por último, en la tarea 8 también pone de manifiesto que recuerda algunos elementos matemáticos con errores

$$
\begin{aligned}
& \text { ¿Cuál es el significado matemático de la Integral } \\
& \text { Definida de la función } y=f(x) \text { en un intervalo } \\
& {[a, b] \text { ? }}
\end{aligned}
$$

En la entrevista el estudiante responde utilizando los argumentos siguientes:

I: ¿Cómo le explicaría a un compañero el significado de la Integral Definida?

A5: Dice que el significado matemático, es que la Integral Definida es un límite y donde el intervalo digámoslo $[a, b]$ está dividido en subintervalos infinitos, los cuales al sumarse en una suma de Riemann nos va a dar un valor numérico.

I: ¿Qué quiere decir en una suma de Riemann y un intervalo infinito?

A5: Una suma de Riemann, es por ejemplo las particiones, o sea, la integral es un número y la suma de Riemann son las particiones que yo puedo hacerle a ese intervalo y sumarlo infinitas veces.

En el protocolo se pone de manifiesto que el estudiante recuerda el elemento matemático ACA cuando menciona las particiones de un intervalo en subintervalos pero lo hace de forma errónea puesto que asociada la subdivisión del intervalo directamente con las sumas de Riemann sin tener en cuenta la función, y además en lugar de considerar infinitos subintervalos habla de subintervalos infinitos Esta puede ser la razón por la que este estudiante no sabe utilizar algunos elementos matemáticos en la resolución de las tareas del cuestionario.

En la siguiente tabla se puede observar las características que mostró este alumno en el desarrollo del esquema del concepto de Integral Definida:

Rev. Invest. Univ. Quindío (21): 95- 107. Armenia - Colombia 
Tabla 1. Relaciones lógicas, elementos matemáticos y sistemas de representación que caracterizan los niveles de desarrollo del esquema de Integral Definida en A5

\begin{tabular}{|c|c|c|c|}
\hline NIVEL & $\begin{array}{l}\text { RELACIONES } \\
\text { LÓGICAS }\end{array}$ & $\begin{array}{c}\text { ELEMENTOS } \\
\text { MATEMÁTICOS }\end{array}$ & $\begin{array}{c}\text { SISTEMAS DE } \\
\text { REPRESENTACIÓN }\end{array}$ \\
\hline INTRA 1 & $\begin{array}{l}\text { No } \\
\text { establecer } \\
\text { relaciones } \\
\text { lógicas } \\
\text { entre los } \\
\text { elementos } \\
\text { matemátic } \\
\text { os. } \\
\text { Recordar } \\
\text { sólo algún } \\
\text { elemento } \\
\text { matemátic } \\
\text { o a lo largo } \\
\text { de todo el } \\
\text { cuestionari } \\
\text { o, } \\
\text { vinculado } \\
\text { sólo a un } \\
\text { sistema de } \\
\text { representa } \\
\text { ción, } \\
\text { gráfico, } \\
\text { algebraico } \\
\text { o analítico. } \\
\text { Recordar } \\
\text { elementos } \\
\text { matemátic } \\
\text { os con } \\
\text { errores. }\end{array}$ & $\begin{array}{l}\text { El área como } \\
\text { aproximación: (G, A) } \\
\text {-Aproximación del área } \\
\text { de una región plana. } \\
\text {-Fórmula del área del } \\
\text { rectángulo. } \\
\text {-Fórmula del área del } \\
\text { triángulo. } \\
\text {-Partición del } \\
\text { intervalo. } \\
\text {-Sumas de Riemann. } \\
\text { El área como límite de } \\
\text { una suma:(AN) } \\
\text {-El límite de las sumas. } \\
\text { La Integral Definida: } \\
\text { (A) } \\
\text {-La definición analítica } \\
\text { de integral definida. } \\
\text {-La integral definida } \\
\text { como área de una } \\
\text { región. } \\
\text { - La integral definida } \\
\text { como cálculo } \\
\text { algebraico } \\
\text {-La integral de } \\
\text { funciones positivas y } \\
\text { negativas. } \\
\text {-Propiedades de la } \\
\text { integral definida de } \\
\text { funciones positivas y } \\
\text { negativas. } \\
\text { Propiedades de la } \\
\text { Integral Definid:(A) } \\
\text {-Integrales especiales. } \\
\text {-De linealidad. } \\
\text { Teoremas } \\
\text { fundamentales:(A) } \\
\text {-Regla de Barrow. }\end{array}$ & $\begin{array}{l}\text { Gráfico } \\
\text { Algebraico } \\
\text { Analítico }\end{array}$ \\
\hline
\end{tabular}

A este alumno se le asigno el subnivel de desarrollo INTRA1 puesto que no es capaz de establecer ninguna relación lógica entre los elementos matemáticos, recuerda los elementos matemáticos de memoria y muestra dificultad para utilizarlos en la resolución de las tareas, utiliza elementos matemáticos sólo en las formas de representación $\mathbf{G}$ y A mostrando algunas concepciones erróneas y resuelve algunas tareas de forma incorrecta.

\section{CONCLUSIONES}

De manera global, por la forma como el alumno resolvió las tareas a lo largo de todo el cuestionario, el modo de justificar las respuestas en la entrevista y la comprensión del concepto de Integral Definida que refleja en el mapa conceptual, podemos afirmar que el alumno recuerda los elementos matemáticos que constituyen el concepto de Integral Definida de memoria y con dificultades, porque los menciona pero no es capaz de aplicarlos en la resolución de las tareas, y cuando intenta utilizarlos además de hacerlo de forma inconexa, muestra concepciones erróneas. No es capaz de manejar el registro G y en el registro A comete muchos errores.

En general, relaciona el concepto de integral con un algoritmo de cálculo, concretamente la utilización de la regla de Barrow, aunque la utilice inadecuadamente en algunas tareas porque no se cumplen las condiciones para aplicarla. No tiene una concepción objeto de la suma de Riemann puesto que recuerda su definición con ciertas concepciones erróneas como las relativas a los subintervalos y, en cuanto a su formulación algebraica, cuando tiene que utilizarla, no sabe cómo hacerlo. No maneja los diferentes sistemas de representación ni los coordina, casi exclusivamente se restringe al sistema algebraico, cierto manejo del gráfico, pero no maneja el registro analítico. No es capaz de aplicar la definición de Integral Definida puesto que confunde las tendencias de la longitud de partición y el número de subintervalos formados por la ausencia de coordinación entre los registros gráfico y algebraico.

Además comete numerosos errores como: errores al calcular las alturas de los rectángulos por no haber síntesis entre los sistemas gráfico y algebraico, confusión en las tendencias de $\mathrm{n}$ y de la longitud de los intervalos, dificultades para expresar una suma de Riemann en una situación concreta, errores en los cálculos de las primitivas.

En definitiva, este alumno utiliza algunos de los elementos matemáticos que constituyen el concepto de Integral Definida desde un pensamiento exclusivamente algebraico y operativo, presenta algunas dificultades con las representaciones gráficas y recuerda de memoria e incluso con errores algunos elementos matemáticos o tiene concepciones erróneas. 


\section{BIBLIOGRAFÍA}

Asiala, M.; Brown, A.; DeVries, D.J.; Dubinsky, E.; Mathews, D ;Thomas, K. (1996). A framework for research and development in ungraduate mathematics education. Research in Collegiate Mathematics Education, 2, 1 32.

Baker, B.; Coolí, L.; Trigueros, M. (2000). A Calculus Graphing Schema. Journal for Research in Mathematics Education, 31(5), $557-578$.

Boigues, F. J. y Pastor, J. (2007). La teoría Fuzzy como elemento para medir el grado de desarrollo en la comprensión de la Integral. En P. Bolea., M. Camacho., P. Flores., B. Gómez., J. Murillo., y M. T. González (Eds.). Investigación en Educación Matemática. Comunicaciones de los grupos de investigación. X Simposio de la SEIEM. Huesca, pp. 145-155.

Calvo, C. (1997). Bases para una Propuesta Didáctica sobre Integrales. Tesis de Maestría. Universitat Autónoma de Barcelona.

Camacho, M., R. Depool y G. Sabrina (2008). Integral Definida en diversos contextos. Un estudio de casos. Educación Matemática, 20(3), 32-57.

Contreras, A. y Ordóñez, L. (2005). Análisis de Significados Personales de los Estudiantes acerca de la Integral Definida. En Maz, A., Gómez, B., y Torralbo, M. (Eds.). Investigación en Educación Matemática. Comunicaciones de los grupos de Investigación. IX Simposio de la SEIEM. Córdoba.

Czarnocha, B.; Loch, S.; Prabhu, V.; Vidakovic, D. (2001). The Concept of definite integral: coordination of two schemas Proceedings of the XXV Conference of the International Group of Mathematics Education, 12 - 17.

Depool, R. A. (2004). La Enseñanza y Aprendizaje del Cálculo Integral en un Entorno Computacional. Actitudes de los Estudiantes Hacia el uso de un Programa de Cálculo Simbólico (PCS). Tesis Doctoral. Universidad de La Laguna.

DeVries, D. J. (2001). RUMEC / APOS Theory Glossary. Georgia Collage \& State University. Milledgeville. http://www.cs.gsu.edu/ rumec/Papers/glossary.html. [Disponible el 18 de agosto de 2008]

Dubinsky, E. (1991). Reflective Abstraction In Advanced Mathematical Thinking, En D. Tall (ed.) Advanced Mathematical Thinking. Dordrecht: Kluwer Academic Press.

Dubinsky, E. (2002a). De la investigación en matemática teórica a la investigación en matemática educativa: un viaje personal. Revista Latinoamericana de investigación en Matemática Educativa., 3 (1), 47 - 70.

Dubinsky, E.; Czarnocha, B.; Loch, S.; Prabhu, Vrunda.; Vidakovic, D. (2000). Conceptions of Area: In Students and in History. College Mathematics Journal. 32 (2), 99-109.

Dubinsky, E.; MacDonald, M.A. (2001). APOS: A Constructivist Theory of Learning in Undergraduated Mathematics Education Research. En D. Holton (eds.). The teaching and Learning of Mathematics at University Level. An ICMI Study. 7 Dordrecht: Kluwer Academia Publisher, 273- 280.

George, A. y Veeramani, P. (1994). On Some Results in Fuzzy Metric Spaces. Fuzzy Sets and Systems, 64, 395399. 
Godino, J. (2002). Un Enfoque Ontológico Semiótico de la Cognición Matemática. Recherches en Didactique des Mathématiques, 22, 2/3, 237-284.

González-Martín, A.S. (2006). La Generalización de la Integral Impropia desde las Perspectivas Numérica, Gráfica y Simbólica Utilizando Entornos Informáticos. Problemas de Enseñanza y Aprendizaje. Tesis Doctoral. Universidad de la Laguna.

Ginsburg, H. P.; Kossan, N. E.; Schwartz, R. \& Swanson, D. (1983). Protocol Methods in Research on Mathematical Thinking. In H. P. Ginsburg (ed.): The Development of Mathematical Thinking. New York: Academic Press.

Mundy, J. (1984). Analysis of Errors of First Year Calculus Students. En A. Bell, B. Low y J. Kilpatrick (eds.). Theory Research and Practice in Mathematics Education. Proceedings, ICME 5. Working group reports and collected papers, Nottingham: Shell Center, 170-172

Orton, A. (1983). Students` Understanding of Integration. Educational Studies in Mathematics. 14, 1- 18.

Paschos, Th. \& Faumak, V. (2006). The reflective abstraction in the construction of the concept of the definite integral. A case study. En J. Novotna; H. Moraova; M. Kretke; N. Stehlikova (eds.) Proceedings of the 30th Conference of Internacional Group for the Psychology of Mathematics Education, 4, 337-344.

Piaget, J; García, R. (1982). Psicogénesis e Historia de la Ciencia. México: Siglo XXI.

Rasslan, S.; TalL, D. (2002). Definitions and Images for the Definite Integral Concept. Proceedings of the 26th PME. 4, 89-96.

Sánchez-Matamoros, G.M. (2004). Análisis de la comprensión en los alumnos de bachillerato y primer año de universidad sobre la noción de derivada (desarrollo del concepto). Tesis Doctoral, Departamento de Didáctica de la Matemática. Universidad de Sevilla.

Turégano, P. (1994). Los Conceptos en Torno a la Medida y el Aprendizaje del Cálculo Infinitesimal, Tesis Doctoral. Universidad de Valencia.

Vinner, S. (1991). The Role of Definitions in the Teaching and Learning of Mathematics. En D. Tall (ed.) Advanced Mathematical Thinking. Dordrecht: Kluwer, p. 65-81. 